\title{
DYNAMIC MUSCULOTENDINOUS TRANSFER TO REPLACE THE ANTERIOR CRUCIATE LIGAMENT IN THE DOG
}

\author{
ROBERT S. ADELAAR, WILLIAM ZUELZER, SCOTT ANTHONY, JOHN A. CARDEA, H. I. LURIE \\ From the Medical College of Virginia, Richmond and Ohio State University, Columbus
}

\begin{abstract}
Dynamic muscle-tendon substitution for acute anterior cruciate deficiency in the dog was studied using the semimembranosus muscle-tendon. Nineteen mongrel dogs each had a semimembranosus transfer in one knee; as a control, the anterior cruciate ligament and the semimembranosus were released in the opposite knee. No postoperative immobilisation was used. The anterior drawer sign was assessed before and after operation and when the dogs were killed five months later. Dogs were excluded from the study if they developed infections or contractures of the hind legs. At five months, 11 dogs were available for study. The operated knees were examined histologically and evaluated using a reproducible index of arthritis based on: the macroscopic discoloration of the articular cartilage, the cellularity of the cartilage, the microscopic appearance of the articular surface, the loss of proteoglycans, the formation of osteophytes and the degree of subchondral osteosclerosis. There was no significant difference in the anterior drawer sign or the degree of arthritic changes between knees with a semimembranous transfer and the controls. Examination showed that a muscle-tendon transfer into the tibia was equivalent to transferring the muscle into the posterior capsulethe intra-articular tendon being weak but histologically viable. The transfer did not prevent the anterior drawer sign becoming positive nor the development of osteoarthritis. A second control group, in which three dogs had an arthrotomy and semimembranosus release in both their hind legs, showed that a semimembranosus release alone did not cause osteoarthritis.
\end{abstract}

Reconstruction of the human anterior cruciate ligament is a complex problem as yet unsolved. The dilemma is whether or not to reconstruct the ligament and, if reconstruction is attempted, which method to use. There are two basic types of reconstruction-static transfer and dynamic transfer. In the static transfer a tenodesis is done to stop the anterior drawer sign, using either the patellar tendon, the iliotibial band, the hamstring tendons or prosthetic materials. The dynamic muscle-tendon transfer, as typified by the gracilis or semitendinosus transfer, substitutes the functioning muscle for the deficient ligament. A method similar to gracilis transfer is described in this experimental study on dogs; the semimembranosus tendon in continuity with its muscle belly, is transferred through the posterior capsule of the knee to the insertion of the anterior cruciate ligament.

The human anterior cruciate is a complex ligament

R. S. Adelaar, MD, Associate Professor of Surgery, Division of Orthopedics

S. Anthony, MD, Clinical Instructor of Surgery, Division of Orthopedics J. A. Cardea, MD, Professor of Surgery, Division of Orthopedics

H. I. Lurie, MD, Professor Emeritus of Pathology, Department of Pathology

Medical College of Virginia, Box 153, MCV Station, Richmond, Virginia 23298, USA.

W. Zuelzer, MD, Department of Orthopedics

Ohio State University, Columbus, Ohio, USA.

Requests for reprints should be sent to Dr R. S. Adelaar.

(C) 1983 British Editorial Society of Bone and Joint Surgery $0301-620 \mathrm{X} / 83 / 5109 \$ 2.00$ with at least two components which act differently depending on the degree of flexion of the knee; no substitute has been developed which can simulate this complex action and there is very little experimental basis for some of the transfers which have been advocated. The object of our study was to test whether, in dogs with anterior cruciate deficiency, our dynamic muscle-tendon transfer modified the development of osteoarthritis. The adult mongrel dog was selected because osteoarthritis can occur naturally and can also be produced by sectioning the anterior cruciate ligament. Osteoarthritis develops rapidly and can be documented histochemically (Lust, Pronsky and Sherman 1972; Ehrlich et al. 1975; Gilbertson 1975; Muir 1977); the degree of degeneration varies with the size and breed of the dog (Nilsson 1948; Paatsama 1952; O'Donoghue et al. 1966, 1971 ; Singleton 1969; Pond and Campbell 1972; Pond and Nuki 1973; Tirgari 1978).

\section{HISTORICAL BACKGROUND}

Möller-Frich was the first to become aware of the problems of anterior cruciate deficiency in English sheepdogs who could no longer work after damaging their anterior cruciate ligaments (Singleton 1969). Paatsama (1952) was the first to describe experimental lesions of the canine knee after division of the anterior cruciate ligament; he found resorption of the anterior cruciate stump, changes in the menisci, degenerative changes of 
the cartilaginous cells and osteophyte formation. Marshall (1969) and Marshall and Olsson (1971) found that three months after the division of the canine anterior cruciate ligament osteophytes formed and continued to grow depending on the instability of the knee, and that there were meniscal tears and degenerative changes in the cartilage. Therefore, the anterior cruciate-deficient dog is a good model for testing substitution procedures. The arthritis that develops after section of the ligament is predictable, occurs in a short period of time and is similar to that which occurs naturally in dogs (Nilsson 1948; Pond and Campbell 1972; Pond and Nuki 1973; Tirgari 1978).

Several methods of evaluating osteoarthritis in dogs have been developed. Some investigators have used Indian ink which becomes trapped in areas where there is a breach in continuity of the surface of the cartilagethe deeper the clefts, the more ink is retained and the darker the stain (Meachim 1972). McDevitt and his colleagues (McDevitt and Muir 1976; McDevitt, Gilbertson and Muir 1977) graded the severity of osteoarthritis on the basis of the size of the area discoloured by the ink and the intensity of the stain. In our opinion this technique offered no more than low-power microscopic examination of the specimen.

Estimation of proteoglycans is another method of evaluating osteoarthritis since more proteoglycans can be extracted from articular cartilage in osteoarthritic joints (Rosenberg 1971; Brandt and Palmoski 1976; McDevitt and Muir 1976; McDevitt et al. 1977). Rosenberg devised a histochemical technique using Safranin O, which binds to charged mucopolysaccharides, to estimate the quantity of proteoglycans in cartilage (Rosenberg 1971; Rosenberg, Pal and Beale 1973). When the Safranin $O$ is applied by Lillie's method, it yields permanent sections in which the concentration of the dye is proportional to the concentration of the mucopolysaccharides.

A system was needed to compare the degree of arthritic changes in different canine joints. McDevitt and his colleagues (1976 and 1977) and Weiss and Mirow (1972) estimated the cellularity of cartilage in sections stained with haematoxylin and eosin and compared degenerative changes in knees at 1, 2, 7, and 16 weeks after the anterior cruciate ligament was sectioned; the lesions were graded by the quality of the articular surface, the loss of proteoglycans and the cellularity. No attempt was made to give a numerical grade to the degree of osteoarthritis and this system only provided a gross estimate that could not be statistically correlated.

It was our feeling that a numerical grading system, using all the above components, would be the ideal form of evaluation and would permit statistical analysis. Our system, which is similar to that developed by Mankin $e t$ al. (1971), is based on histological and histochemical examination of fragments of articular cartilage. Our grading system gives a better index of the severity of osteoarthritis of the whole joint and an index which can be used for comparison with degenerative changes in other joints.

\section{MATERIALS AND METHOD}

Nineteen conditioned adult mongrel dogs, weighing an average of 24 kilograms (range 17 to 35.5 kilograms), were anaesthetised with intravenous sodium pentobarbital ( 0.5 millilitres per kilogram of body weight). The knees were tested for the presence of the anterior drawer sign. Both hind legs were shaved, then scrubbed with Betadine solution. Alternate left and right knees were used for tendon transfer, the other leg acting as a control. A medial parapatellar incision was used to open the knee and expose the semimembranosus muscle-tendon unit, which is more distal in the dog than in man (Fig. 1). The surfaces of the joint were inspected for any evidence of degenerative disease, and the anterior cruciate ligament carefully sectioned and removed, taking care not to damage the closely adjacent insertion of the posterior cruciate ligament. The semimembranosus was detached at its insertion and the tendon dissected free from all fascial attachments to the level of the mid-thigh (Fig. 1).

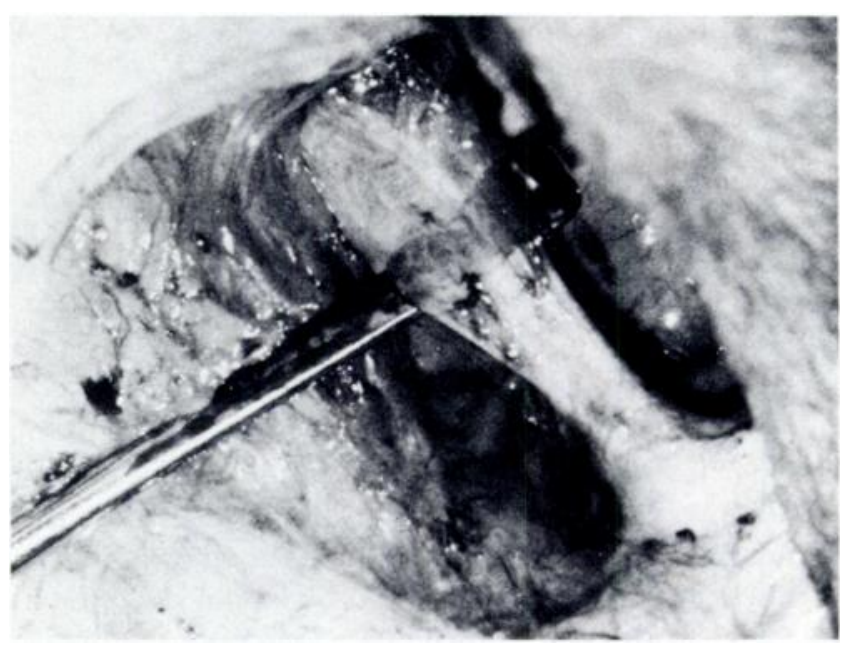

Fig. 1

Photograph showing the insertion of the semimembranosus tendon; this is lower in dogs than in humans.

The semimembranosus was then passed through a hole in the posterior capsule lateral to the posterior cruciate ligament (Fig. 2) and attached with a Bunnell wire suture at the site of insertion of the anterior cruciate. It was attached to the soft tissue and periosteum of the anterior tibia with sufficient tension so that Blix optimum tension criteria could be satisfied. The tendon was stretched to its maximal tension and sutured at half the distance of its resting length. On the opposite control knee, the same operative approach was used and the anterior cruciate ligament and semimembranosus were released. In a second control group arthrotomy and semimembranosus 
release was performed on both hind legs of three dogs (six knees) to see if this alone caused arthritis.

At the end of each operation the knees were tested for the presence of the anterior drawer sign in neutral rotation and 45 degrees of flexion. The dogs were not immobilised after operation as this has been reported to lead to osteoarthritis (Akeson, Amiel and La Violette 1967; Langenskiöld, Michelsson and Videman 1979). Each dog was kept in a single cage for the first 7 to 10 days in order to make sure that they were walking equally on both hind legs before they were transferred to a large run at a protected farm. Serial radiographs were taken to ensure that the Bunnell-wired tendon had not become detached.

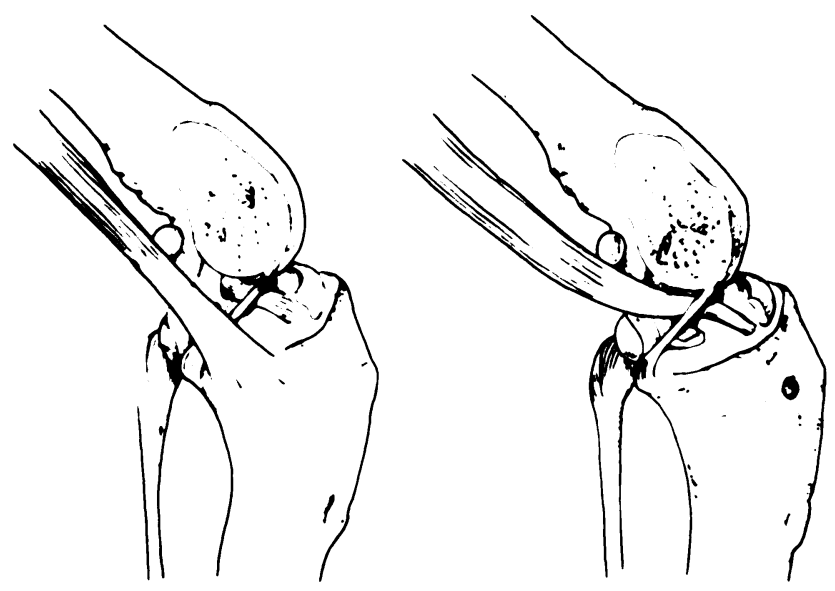

Fig. 2

Diagrams showing the semimembranosus passed through a hole in the posterior capsule lateral to the posterior cruciate and attached at the site of the anterior cruciate insertion.

The animals were killed if there was any evidence of infection or contracture which would preclude full symmetrical function of the hind legs. After five months 11 dogs were available for study, plus the three dogs that comprised the second control group. The anterior drawer sign was measured clinically and by lateral radiographs in the anaesthetised animal. The dogs were then killed and the knees dissected and examined to see whether pulling the semimembranosus insertion through to the posterior capsule had eliminated the drawer sign. The intra-articular portion of the tendon was evaluated histologically. The knees were removed from the dogs, assigned a random number and sent to the pathologist (HL).

The pathologist evaluated each specimen without knowledge of which procedure had been performed and graded it according to the degree of degenerative changes using the arthritic index illustrated in Figures 3 to 10. The articular surfaces of the femoral condyles and tibial plateaux were inspected with the naked eye and with the use of a $\times 7$ hand-lens. The areas were inspected for discoloration (pale yellow to brown), fibrillation, and the presence of osteophytes. The specimens were fixed in a
10 per cent buffered formalin solution. The articular surface and a small portion of underlying bone were removed and decalcified in a solution of sodium citrate and formic acid. The specimen blocks were embedded in paraffin wax and sectioned into layers six to eight microns thick. The sections were stained with haematoxylin and eosin, aldehyde fuchsine and Safranin $O$.

The sections were studied microscopically and, based on the arthritic index developed by Lurie, values were assigned to each of the following: the discoloration of the articular cartilage (Fig. 3), the integrity and the cellularity of the articular cartilage (Figs 4 and 5), the formation of osteophytes (Fig. 6), the loss of proteoglycans (Figs 7 and 8), and the extent of subchondral osteosclerosis (Figs 9 and 10). A maximal value of 68 points with 34 for the femur and 34 for the tibia could be obtained for severe degenerative changes.

\section{RESULTS}

Eleven of the 19 dogs that underwent replacement of the anterior cruciate ligament were found to qualify for postoperative analysis. The rest were eliminated because of infection, contracture of the hind legs or other conditions such as parvovirus which was prevalent during the period of the experiment.

Before operation none of the dogs had an anterior drawer sign of greater than one millimetre and the findings immediately after operation were not significantly different. After five months the average measurement of the anterior drawer sign for the transferred intact joint was 3 millimetres and for the opposite control joint was 3.1 millimetres; this difference between the two was not significant using the independent paired $t$ test. The anterior drawer sign in the control group with arthrotomy and a semimembranosus release was the same before and after operation.

The index used to evaluate the degree of osteoarthritis is illustrated in Figures 3 to 10. The difference in the arthritic index of the joints that had a semimembranosus transfer and the control knees that had an anterior cruciate and semimembranosus release was not significant using the independent paired $t$ test $(P>0.98)$. However, there was a significant difference between the control group with arthrotomy and the transfer group, and between the arthrotomy group and the anteriorcruciate-release control group $(P<0.01)$. The arthrotomy control group had statistically less microscopic arthritic changes than either of the other groups.

Examination of the tendon of the semimembranosus in knees that had undergone transfer revealed that it was uniformly larger than before transfer, but it had no significant structural integrity and appeared to be loose when probed; therefore, no Instron testing could be done. Histological examination of four specimens of transferred semimembranosus showed that the tendon was viable and had not undergone fibrous transformation or necrosis. 
Figure 3-The coloration and loss of translucency of cartilage is graded from zero to four: $0=$ normal; $2=$ yellow and slight loss of translucency; $4=$ brown and moderate loss of translucency. Figure 4-The integrity of the cartilage is evaluated histologically and graded from zero to eight: $0=$ normal; 2 = roughening or $\mathrm{f}$ brillation of surface restricted to the tangential zone: $4=$ clefts extending into the transitional zone; $6=$ clefts extending into radial zone or breach of tidemark or both; $8=$ loss of cartilage.

Figure 5-The cellularity of the cartilage is graded from zero to six: $0=$ normal; $1=$ occasional cell doublets; $2=$ small clones in the transitional zone; $3=$ generalised increase in cellularity: $4=$ large clones extending into the radial zone; $6=$ loss of cells. Figure 6 Osteophyte formation is graded from zero to six: $0=$ no evidence of osteophytes: $2=$ microscopic evidence of early osteophyte formation; 3 = fully formed osteophytes visible under the microscope; 4 = small osteophytes visible to the naked eye: $6=$ numerous large osteophytes.

Figures 7 and 8-The proteoglycan content evaluated by staining with Safranin $O$ is graded from zero to six: $0=$ normal; $\quad 2=$ decreased staining in the transitional zone: $\mathbf{4}=$ decreased staining in the radial zone; $6=$ absence of staining in all zones.

Figures 9 and $10-$ Subchondral sclerosis is evaluated from zero to four in sections stained with haematoxylin and eosin: $0=$ no evidence $; 2=$ focal $; 4=$ diffuse

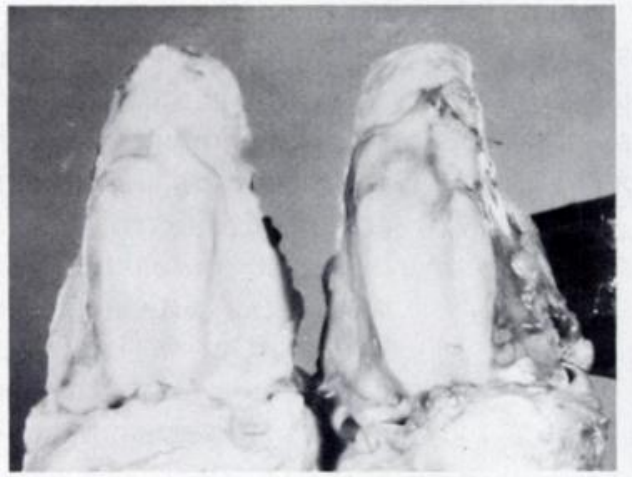

Fig. 3

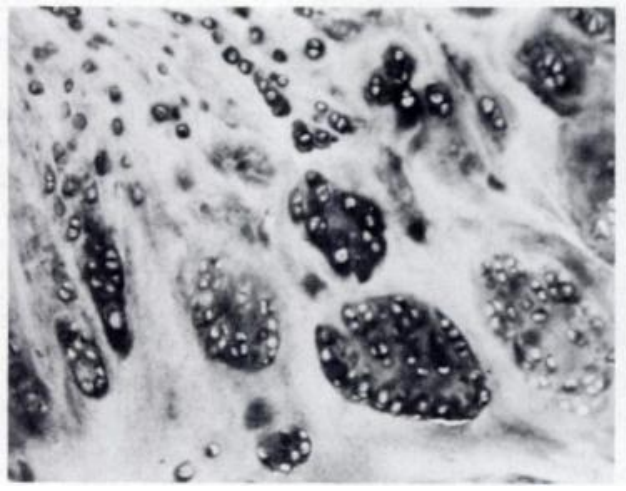

Fig. 5

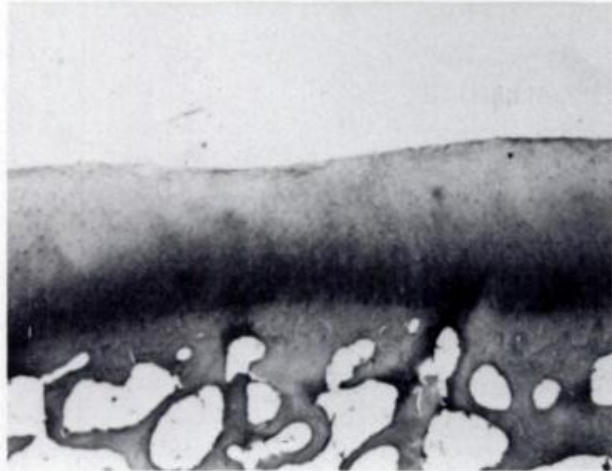

Fig. 7
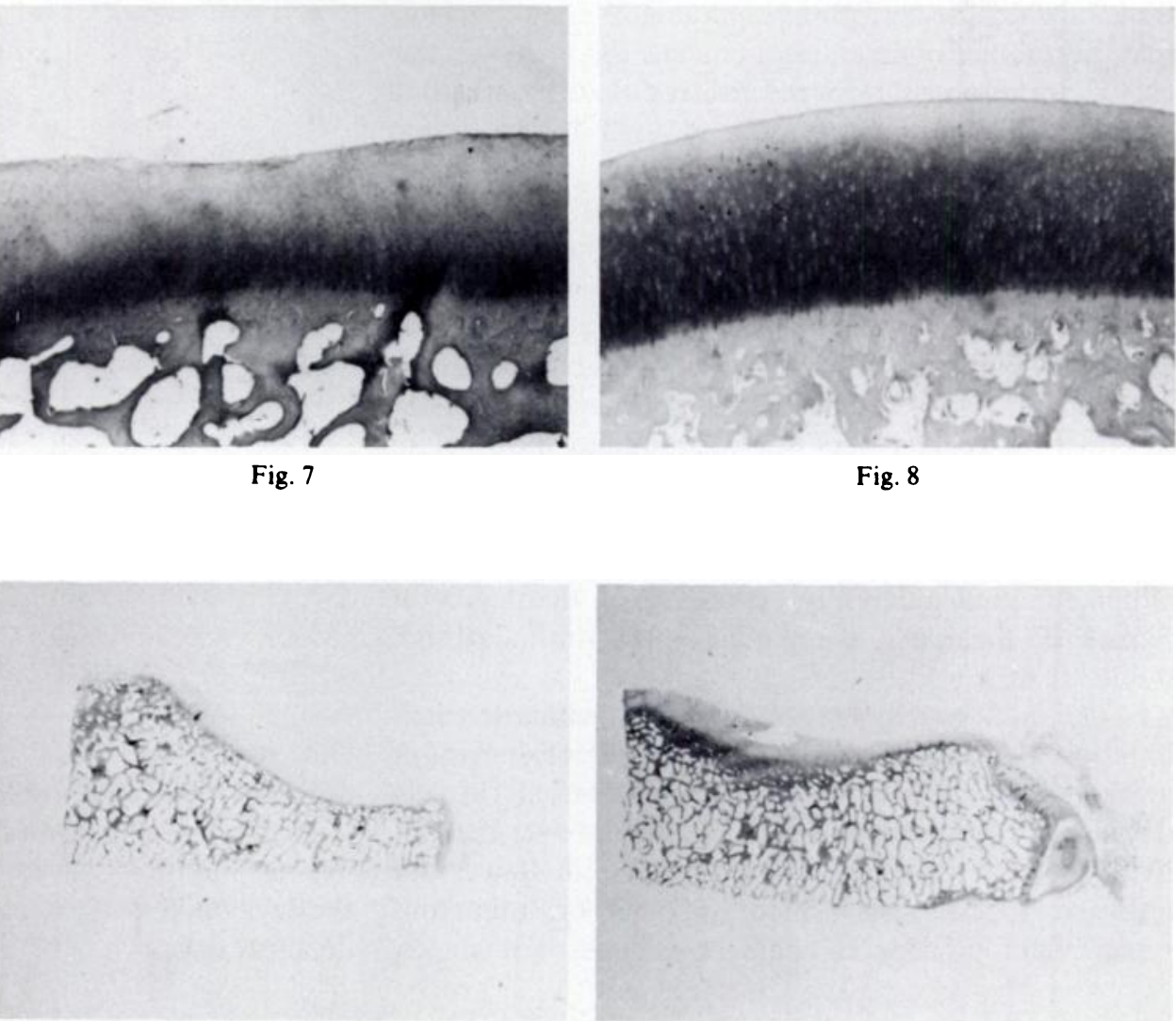

Fig. 9
Fig. 8

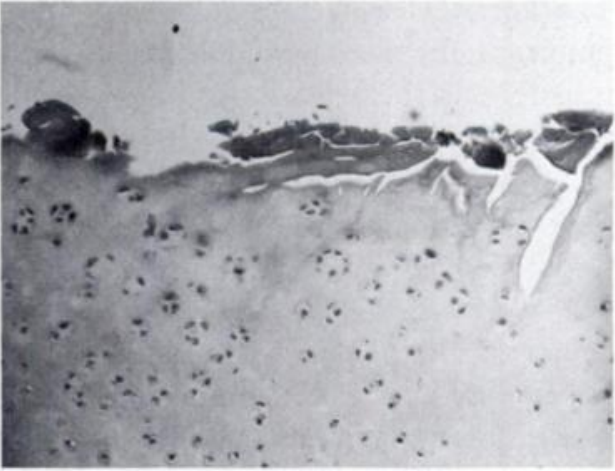

Fig. 4

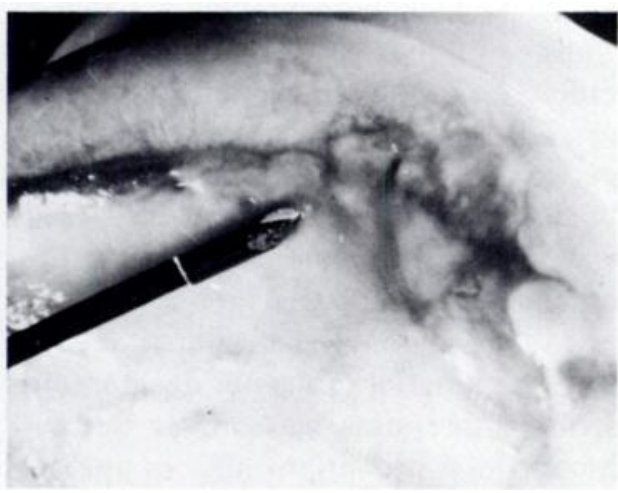

Fig. 6

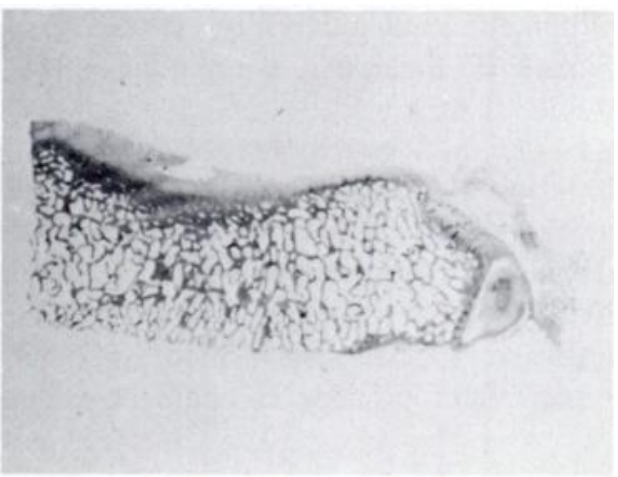

Fig. 10 
The anterior drawer test could not be eliminated by pulling on the muscle-tendon/posterior-capsule unit. The transfer into the posterior capsule was found to be solidly attached but this did not stop the anterior drawer sign from occurring in the dead animal. It should be noted that our transfer is a dynamic one, but in our study we did not evaluate the dynamic effect on the posterior capsule in the unanaesthetised animal.

\section{DISCUSSION}

The results of our study in dogs showed that there was no statistical difference in the development of osteoarthritis five months after operation between knees in which the cruciate and semimembranosus had been sectioned and knees in which this procedure was followed by a dynamic transfer of the semimembranosus; nor was there any difference in the clinical anterior drawer test in neutral rotation between the two groups. The intra-articular portion of the semimembranosus was found to be viable but not functional; its attachment to the posterior capsule was firm, but this did not decrease the arthritis in the cruciate-deficient dog. These results confirm our hypothesis that dynamic transfers are not adequate substitutes for the anterior cruciate ligament in the dog. The semimembranosus was chosen since it was the only muscle of appropriate size in the mongrel dog and resection of the semimembranosus alone did not cause degenerative changes. The Lindemann transfer uses the gracilis, but the principles are exactly the same as the transfer which we performed in the dog. We can conclude that the absence of an anterior cruciate ligament was the only factor responsible for the arthritic changes, and that a dynamic hamstring transfer through the posterior capsule did not prevent its development.

Based on this work and on similar experiments currently being conducted using static transfers (such as the iliotibial band and patellar tendon) it is our feeling that too many procedures for replacing the anterior cruciate ligament are being used without adequate experimentation to determine their efficacy. If a transfer or prosthetic replacement is to function like the cruciate ligament, it will need to have a tenodesis effect to prevent the anterior drawer sign. The human anterior cruciate ligament has at least two separate major components which function differently depending on the degree of flexion of the knee; a one-phase substitution cannot duplicate this.

Our main goal in treating the human with a deficient anterior cruciate ligament is to stop the development of arthritis. It has been our observation that patients usually develop radiographic evidence of arthritis over the first five years after the ligament has ruptured. It was our objective to establish a model so that the numerous replacement procedures could be tried experimentally before being used clinically. In spite of the fact that the transfers appear solid when put in, we question their ability to replace the cruciate effectively.

A comprehensive arthritic index was developed which can be used in future experiments to compare arthritis in different knees. Our index differs from others based on histological and histochemical grading of sections of articular cartilage (Mankin et al. 1971; McDevitt et al. 1977); in these systems, the grading varies enormously from section to section and is not effective in assessing the severity of osteoarthritis in the joint as a whole. Using our method we found that there was a statistical correlation of 0.05 or better between discoloration or loss of translucency of cartilage, its cellular integrity, the loss of proteoglycans, the presence of subchondral sclerosis and the formation of osteophytes.

Table I. Scores for arthritis in knees with a semimembranosus transfer, knees with anterior cruciate release and knees with arthrotomy and semimembranosus release

\begin{tabular}{|c|c|c|c|}
\hline \multirow[b]{3}{*}{ Dog number } & \multicolumn{3}{|c|}{ Arthritic index (maximum score $=68$ ) } \\
\hline & \multirow[b]{2}{*}{$\begin{array}{l}\text { Dynamic } \\
\text { transfer }\end{array}$} & \multicolumn{2}{|l|}{ Controls } \\
\hline & & $\begin{array}{l}\text { Anterior } \\
\text { cruciate release }\end{array}$ & $\begin{array}{l}\text { Arthrotomy and } \\
\text { semimembranosus } \\
\text { release }\end{array}$ \\
\hline 1 & 15 & 17 & - \\
\hline 2 & 29 & 18 & - \\
\hline 3 & 41 & 50 & - \\
\hline 4 & 43 & 43 & - \\
\hline 5 & 42 & 54 & - \\
\hline 6 & 39 & 28 & - \\
\hline 7 & 34 & 30 & - \\
\hline 8 & 27 & 27 & - \\
\hline 9 & 17 & 18 & - \\
\hline 10 & 37 & 42 & - \\
\hline 11 & 48 & 44 & - \\
\hline 12 & - & - & $\begin{array}{r}18 \\
6\end{array}$ \\
\hline 13 & - & - & $\begin{array}{l}16 \\
18\end{array}$ \\
\hline 14 & - & - & $\begin{array}{l}17 \\
17\end{array}$ \\
\hline Average & 33.8 & 33.7 & 15 \\
\hline
\end{tabular}

Our numerical index allowed comparisons between different joints and procedures, and our figures (Table I) showed a very narrow range of values for each procedure. We feel that this is a very sensitive method of evaluating the development of osteoarthritis in the anterior cruciatedeficient dog. 


\section{REFERENCES}

Akeson WH, Amiel D, La Violette D. The connective tissue response to immobility: a study of the chondroitin-4 and 6-sulfate and dermatan sulfate changes in periarticular connective tissue of control and immobilized knees of dogs. Clin Orthop 1967;51:183-97.

Brandt KD, Palmoski M. Organization of ground substance proteoglycans in normal and osteoarthritic knee cartilage. Arthritis Rheum 1976; 19:209-15.

Ehrlich MG, Mankin HJ, Jones H, Grossman A, Crispen C, Ancona D. Biochemical confirmation of an experimental osteoarthritis model. J Bone Joint Surg [Am] 1975;57-A:392-6.

Gilbertson E. Development of periarticular osteophytes in experimentally induced osteoarthritis in the dog. Ann Rheum Dis 1975;34:12-25.

Langenskiöld A, Michelsson J-E, Videman T. Osteoarthritis of the knee in the rabbit produced by immobilization. Acta Orthop Scand 1979;50: $1-14$.

Lust G, Pronsky W, Sherman DM. Biochemical and ultrastructural observations in normal and degenerate canine articular cartilage. Am J Vet Res 1972;33:2429-40.

McDevitt CA, Muir H. Biochemical changes in the cartilage of the knee in experimental and natural osteoarthritis in the dog. J Bone Joint Surg $[B r] 1976 ; 58-B: 94-101$.

McDevitt C, Gilbertson E, Muir H. An experimental model of osteoarthritis: early morphological and biochemical changes. J Bone Joint Surg [Br] 1977;59-B:24-35.

Mankin HJ, Dorfman H, Lippiello L, Zarins. Biochemical and metabolic abnormalities in articular cartilage from osteo-arthritic human hips. II : correlation of morphology with biochemical and metabolic data. J Bone Joint Surg [Am] 1971;53-A:523-37.

Marshall JL. Periarticular osteophytes: initiation and formation in the knee of the dog. Clin Orthop 1969;62:37-47.

Marshall JL, Olsson S-E. Instability of the knee: a long-term experimental study in dogs. J Bone Joint Surg [Am] 1971;53-A : 1561-70.

Meachim G. Light microscopy of India ink preparations of fibrillated cartilage. Ann Rheum Dis 1972;31:457-64.

Muir H. Molecular approach to the understanding of osteoarthrosis. Ann Rheum Dis 1977;36:199-208.

Nilsson F. Meniscal injuries in dogs. North Am Vet 1949;30:509-15.

O'Donoghue DH, Frank GR, Jeter GL, Johnson W, Zeiders JW, Kenyon R. Repair and reconstruction of the anterior cruciate ligament in dogs: factors influencing long-term results. J Bone Joint Surg [Am] 1971;53-A:710-8.

O'Donoghue DH, Rockwood CA Jr, Frank GR, Jack SC, Kenyon R. Repair of the anterior cruciate ligament in dogs. J Bone Joint Surg [Am] $1966 ; 48-A: 503-19$.

Paatsama S. Ligament injuries in the canine stifle joint : a clinical and experimental study. Helsinki Conference, 1952.

Pond MJ, Campbell JR. The canine stifle joint. I: rupture of the anterior cruciate ligament: an assessment of conservative and surgical treatment. J Small Anim Pract 1972;13:1-10.

Pond MJ, Nuki G. Experimentally-induced osteoarthritis in the dog. Ann Rheum Dis 1973;32:387-8.

Rosenberg L. Chemical basis for the histological use of Safranin O in the study of articular cartilage. J Bone Joint Surg [Am] 1971;53-A:69-82.

Rosenberg LC, Pal S, Beale RJ. Proteoglycans from bovine proximal humeral articular cartilage. J Biol Chem 1973;248:3681-90.

Singleton WB. Observations based upon the surgical repair of 106 cases of anterior cruciate ligament rupture. J Small Anim Pract 1969;10: 269-78.

Tirgari M. Changes in the canine stifle joint following rupture of the anterior cruciate ligament. J Small Anim Pract 1978;19:17-26.

Weiss C, Mirow S. An ultrastructural study of osteoarthritic changes in the articular cartilage of human knees. J Bone Joint Surg [Am] 1972; 54-A:954-72. 